Revue des sciences de l'eau

\title{
Étude de la performance du milieu filtrant constitué d'une couche de chènevotte sur une couche de grignons d'olives dans le traitement des eaux usées domestiques : du pilote à l'échelle industrielle \\ Study of the performance of a filter medium consisting of a layer of hemp shives on a layer of olive pomace in the treatment of domestic wastewater: scale-up from biofiltration pilot to full-scale
}

Jesús Villalobos Garcia, Claire Vialle, Caroline Sablayrolles, Mireille Montrejaud-Vignoles, Jean-Philippe Amalric, Matthias Desmolles, Christian Vignoles, Patrice Gallien et Claire Albasi

Volume 32, numéro 1, 2019

Reçu le le 30 mai 2018, accepté le 15 septembre 2018

URI : https://id.erudit.org/iderudit/1059876ar

DOI : https://doi.org/10.7202/1059876ar

Aller au sommaire du numéro

Éditeur(s)

Université du Québec - INRS-Eau, Terre et Environnement (INRS-ETE)

ISSN

1718-8598 (numérique)

Découvrir la revue

Citer cet article

Garcia, J. V., Vialle, C., Sablayrolles, C., Montrejaud-Vignoles, M., Amalric, J.-P., Desmolles, M., Vignoles, C., Gallien, P. \& Albasi, C. (2019). Étude de la performance du milieu filtrant constitué d'une couche de chènevotte sur une couche de grignons d'olives dans le traitement des eaux usées domestiques : du pilote à l'échelle industrielle. Revue des sciences de l'eau / Journal of Water Science, 32(1), 1-10. https://doi.org/10.7202/1059876ar

\section{Résumé de l'article}

Deux matériaux, sous-produits agro-industriels, ont été étudiés en biofiltration à l'échelle pilote : la chènevotte en tant que " couche active ", sur des grignons d'olives en tant que " couche de support ». L'objectif était d'étudier la capacité de ces deux matériaux travaillant ensemble comme un seul milieu filtrant poreux. L'impact de la hauteur de la couche active sur la performance du biofiltre a été étudié expérimentalement grâce à deux biofiltres, un premier présentant une répartition des matériaux en deux hauteurs égales, l'autre avec $80 \%$ de couche active sur $20 \%$ de hauteur de couche support. Les deux pilotes ont été alimentés à raison de $75 \mathrm{~L} \cdot \mathrm{j}^{-1} \cdot \mathrm{EH}^{-1}$ (EH : équivalent-habitant) avec des eaux usées domestiques synthétiques. Les résultats ont montré une réduction de la demande biochimique en oxygène en cinq jours $\left(\mathrm{DBO}_{5}\right)$ et des matières en suspension (MES) de plus de $90 \%$ après dix semaines de fonctionnement. Cette performance s'est maintenue après plus de 30 semaines de fonctionnement continu. Par ailleurs, six prototypes de biofiltres ont été installés pour traiter les eaux usées domestiques de résidences unifamiliales dans des conditions réelles. Les objectifs de cette étude sont de caractériser l'efficacité du traitement de ces prototypes sur plusieurs années dans des conditions réelles, de déterminer l'entretien requis par une telle technologie et d'étudier l'influence de paramètres tels que la hauteur du biofiltre, l'aération et le nombre d'habitants. L'efficacité de traitement à long terme de l'un des biofiltres, qui fonctionne depuis près de quatre ans, est remarquable. Les pourcentages moyens de réduction de la demande chimique en oxygène (DCO), de la $\mathrm{DBO}_{5}$ et de MES sont respectivement de plus de $83 \%$, $97 \%$ et $96 \%$, laissant espérer des résultats prometteurs pour les cinq autres. 


\section{ÉTUDE DE LA PERFORMANCE DU MILIEU FILTRANT CONSTITUÉ D’UNE COUCHE DE CHĖNEVOTTE SUR UNE COUCHE DE GRIGNONS D'OLIVES DANS LE TRAITEMENT DES EAUX USÉES DOMESTIQUES : DU PILOTE À L'ÉCHELLE INDUSTRIELLE}

Study of the performance of a filter medium consisting of a layer of hemp shives on a layer of olive pomace in the treatment of domestic wastewater: scale-up from biofiltration pilot to full-scale

Jesús VILLALOBOS GARCLA 1,2,3*, CLAIRE VIALLE², CAROLINE SABLAYROLLES ${ }^{2}$, MiREILLE MONTREJAUD-

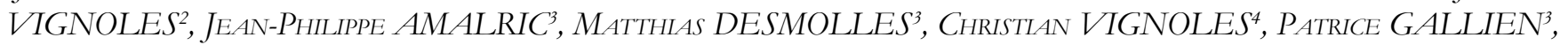

CLAIRE ALBASI

${ }^{1}$ Laboratoire de Génie Chimique (LGC), Université de Toulouse, CNRS, INPT, UPS, Toulouse, France

${ }^{2}$ Laboratoire de Chimie Agro-industrielle (LCA), Université de Toulouse, INRA, INPT, Toulouse, France

${ }^{3}$ Société ITREN, route Dieupentale, 82600 Verdun-sur-Garonne, France

${ }^{4}$ Assainissement Vignoles Consulting SAS, 36 rue Velasquez, 31300 Toulouse, France

Reçu le le 30 mai 2018, accepté le 15 septembre 2018

\section{RÉSUMÉ}

Deux matériaux, sous-produits agro-industriels, ont été étudiés en biofiltration à l'échelle pilote : la chènevotte en tant que " couche active ", sur des grignons d'olives en tant que " couche de support ». L'objectif était d'étudier la capacité de ces deux matériaux travaillant ensemble comme un seul milieu filtrant poreux. L'impact de la hauteur de la couche active sur la performance du biofiltre a été étudié expérimentalement grâce à deux biofiltres, un premier présentant une répartition des matériaux en deux hauteurs égales, l'autre avec $80 \%$ de couche active sur $20 \%$ de hauteur de couche support. Les deux pilotes ont été alimentés à raison de $75 \mathrm{~L} \cdot \mathrm{j}^{-1} \cdot \mathrm{EH}^{-1}$
(EH : équivalent-habitant) avec des eaux usées domestiques synthétiques. Les résultats ont montré une réduction de la demande biochimique en oxygène en cinq jours $\left(\mathrm{DBO}_{5}\right)$ et des matières en suspension (MES) de plus de $90 \%$ après dix semaines de fonctionnement. Cette performance s'est maintenue après plus de 30 semaines de fonctionnement continu. Par ailleurs, six prototypes de biofiltres ont été installés pour traiter les eaux usées domestiques de résidences unifamiliales dans des conditions réelles. Les objectifs de cette étude sont de caractériser l'efficacité du traitement de ces prototypes sur plusieurs années dans des conditions réelles, de déterminer l'entretien requis par une telle technologie et d'étudier l'influence de paramètres tels que la hauteur du 
biofiltre, l'aération et le nombre d'habitants. L'efficacité de traitement à long terme de l'un des biofiltres, qui fonctionne depuis près de quatre ans, est remarquable. Les pourcentages moyens de réduction de la demande chimique en oxygène (DCO), de la $\mathrm{DBO}_{5}$ et de MES sont respectivement de plus de $83 \%, 97 \%$ et $96 \%$, laissant espérer des résultats prometteurs pour les cinq autres.

Mots-clés : traitement des eaux usées, milieu filtrant, biomasse, biofiltration, valorisation.

\section{ABSTRACT}

Two agro-industrial by-products were studied in biofiltration of domestic wastewater at pilot scale: hemp shives working as "active layer", or top layer, on olive pomace working as "support layer", or bottom layer. The aim of this work was to study the treatment capacity of these two materials working together as one single porous filter medium. The first biofilter had an equal proportion of both materials while the second one had $80 \%$ of the active layer and $20 \%$ of the support layer. The objective was to study the impact of the layer height on the biofilter performance. Both pilots were fed with synthetic sludge-based wastewater at a rate of $75 \mathrm{~L} \cdot \mathrm{d}^{-1} \cdot \mathrm{PE}^{-1}$ (PE: population equivalent). Results showed a reduction of more than $90 \%$ of the biochemical oxygen demand at five days $\left(\mathrm{BOD}_{5}\right)$ and the total suspended solids (TSS) after ten weeks of continuous operation. The performance remains good after more than 30 weeks of operation. Since the laboratory results were very encouraging, six prototype biofilters were installed to treat domestic wastewater from single-family residences under real conditions. The short-term purposes of this study are to determine the maintenance required by such a novel technology and to study the influence of parameters such as filter height, aeration, and the number of inhabitants. While the long-term goal is to characterize the treatment efficiency of these prototypes over many years under field conditions. The long-term treatment efficiency of one of the biofilters, which has been running for almost four years, is outstanding. The mean reduction in the chemical oxygen demand (COD), $\mathrm{BOD}_{5}$, and TSS is over $83 \%, 97 \%$, and $96 \%$ respectively, rising expectations for the other five prototypes.

Key words: wastewater treatment, filter media, biomass, biofiltration, valorization.

\section{INTRODUCTION}

L'assainissement a de tout temps été une préoccupation majeure pour des questions d'hygiène et de santé publique. Le but de l'assainissement est de fournir un environnement de vie sain à tout être vivant, en éloignant ou éliminant les excréments, pour éviter le contact avec la population, limitant ainsi les épidémies (OMS/UNICEF, 2013). Une installation d'assainissement non collectif (ANC) est définie comme " toute installation d'assainissement assurant la collecte, le transport, le traitement et l'évacuation des eaux usées domestiques ou assimilées au titre de l'article R. 214-5 du code de l'environnement des immeubles ou parties d'immeubles non raccordés à un réseau public de collecte des eaux usées " (MEEDDM, 2009). En France, l'ANC représente $20 \%$ des installations de traitement des eaux usées domestiques. Cela concerne une population de 12 millions d'habitants, soit environ cinq millions d'installations en zones rurales (DUBOIS et BOUTIN, 2017).

Parmi les technologies utilisées dans l'ANC, les systèmes de filtration biologique utilisant un milieu filtrant sont souvent mis en œuvre (GARZON-ZUNIGA et al., 2012; LENS et al., 1994). Le traitement des eaux usées (élimination des matières en suspension et de la matière organique) est effectué par les actions conjointes de filtration, et de biodégradation par des bactéries se développant au sein du milieu filtrant. L'aération des milieux s'effectue naturellement avec une forte dépendance à la porosité et à l'agencement du matériau (BERLAND, 2012; CAUCHI et VIGNOLES, 2011).

Historiquement, des matériaux comme le sable et la tourbe sont les plus utilisés, et plus récemment des milieux à base de copeaux de coco venus remplacer la tourbe dont l'extraction est interdite, sont en développement croissant. Cependant, l'empreinte carbone produite par l'importation depuis l'étranger de matériaux tels que ce dernier est très importante. Dès lors l'utilisation de sous-produits agro-industriels locaux pourrait la diminuer et rendre l'utilisation de ces nouveaux milieux filtrants plus compatible avec des critères environnementaux.

Pour ce faire, la société ITREN, le Laboratoire de génie chimique et le Laboratoire de chimie agro-industrielle développent ensemble un nouveau dispositif de traitement décentralisé (GALLIEN et al., 2016) : un milieu filtrant constitué d'une couche de chènevotte sur une couche de grignons d'olives, testé d'abord à l'échelle pilote puis à l'échelle réelle. L'innovation réside, entre autres, dans la valorisation de ces sous-produits agro-industriels disponibles localement. Lobjectif de cet article est de vérifier la capacité de ce milieu filtrant à traiter les eaux usées domestiques. Les résultats du suivi de la qualité physico-chimique des eaux rejetées en sortie de pilotes laboratoire et sur le terrain sont présentés. 


\section{MATÉRIELS ET MÉTHODES}

\subsection{Pilotes de biofiltration au laboratoire}

Quatre unités-pilotes à l'échelle du laboratoire en PVC transparent ont été mises en œuvre (Figure 1). Leur conception est basée sur des travaux de recherche antérieurs (BRETON, 2013). Un pilote avec des copeaux de coco (Premier Tech Aqua, Canada) est utilisé comme référence. Deux pilotes ont été remplis avec une couche de chènevotte (Chanvrière de l'Aube, Bar-sur-Aube, France) sur une couche des grignons d'olives (Établissement Bardon Lignoblast, Le Muy, France) avec des hauteurs identiques. Ces deux pilotes similaires permettront d'évaluer la reproductibilité et la robustesse des résultats. Un dernier pilote avec une hauteur plus importante de chènevotte (80\%) permet d'étudier l'impact de la hauteur des couches sur la performance épuratrice des biofiltres.

Une solution synthétique, réalisée au laboratoire à base d'une boue digérée récupérée en sortie de digesteur complétée par des composés chimiques pour obtenir une DCO maitrisée, alimente par gravité l'ensemble des pilotes à raison de $75 \mathrm{~L} \cdot \mathrm{j}^{-1} \cdot \mathrm{EH}^{-1}$ (EH : équivalent-habitant). Cette solution permet d'assurer des valeurs d'entrée des matières en suspension $\left(\mathrm{MES} \approx 170 \mathrm{mg} \cdot \mathrm{L}^{-1}\right.$ ), de demande biochimique en oxygène en cinq jours $\left(\mathrm{DBO}_{5} \approx 300 \mathrm{mg} \mathrm{O} \cdot \mathrm{L}^{-1}\right)$ et de demande chimique en oxygène $\left(\mathrm{DCO} \approx 800 \mathrm{mg} \mathrm{O} \mathrm{O}_{2} \cdot \mathrm{L}^{-1}\right)$ constantes. Le $\mathrm{pH}$ moyen de cette solution est de 7,2.

Un suivi hebdomadaire des eaux collectées en sortie des pilotes sur $24 \mathrm{~h}$ permet de suivre l'évolution de l'épuration. Les méthodes analytiques utilisées sont les suivantes : DCO : ISO 15705 (tests rapides Spectroquant ${ }^{\oplus}$ Merck; ISO, 2002); $\mathrm{DBO}_{5}$ : NF EN 1899-1 (AFNOR, 1998); MES : NF EN 872 (AFNOR, 2005); pH : NF T 90-008 (AFNOR, 2001).

\subsection{Prototypes terrains}

Six prototypes de biofiltre ont été installés chez des particuliers pour étudier leurs performances en conditions réelles. Les familles ont été choisies en fonction de leur localisation, du nombre d'habitants, et de leur disposition à faire partie de cette étude. La figure 2 résume les conditions opératoires de l'étude pour chaque site : composition des familles, date de la mise en service, proportion des couches chènevotte sur grignons d'olives (50:50 ou 80:20). De plus, pour étudier les effets de l'aération, six tubes de diamètre $50 \mathrm{~mm}$, ont été répartis dans chaque cuve. Ces tubes peuvent être percés (16 orifices de $5 \mathrm{~mm}$ uniformément répartis) ou pleins.
Les cuves sont en béton fibré (fibres en polyéthylène), avec un volume utile du système de traitement de $6000 \mathrm{~L}$ pour le site A et $5400 \mathrm{~L}$ pour les autres sites. La hauteur de milieu filtrant est fixée à $80 \mathrm{~cm}$. Le suivi a été réalisé de façon périodique, avec une campagne de prélèvements mensuelle. Les prélèvements en sortie du biofiltre sont réalisés à raison d'un prélèvement moyen sur $24 \mathrm{~h}$. Les teneurs moyennes en DCO, MES et $\mathrm{DBO}_{5}$ observées dans la littérature (CHABAUD, 2007) pour les eaux usées prétraitées (EUPT), eaux issues du prétraitement des filières d'ANC, sont utilisées comme valeurs théoriques de référence en entrée du biofiltre pour calculer les taux d'abattement.

\section{RÉSULTATS ET DISCUSSION}

\subsection{Etude pilote en laboratoire}

L'évolution des différents paramètres suivis pendant plus de 35 semaines d'opération ininterrompue est présentée à la figure 3.

Pour faciliter les comparaisons entre pilotes, le numéro de la semaine correspond au temps qui s'est écoulé à partir de la mise en service du pilote. Létude a commencé à la dixième semaine, temps préconisé pour l'établissement de la biomasse épuratrice. La période comprise entre les semaines 24 et 28 correspond à la fermeture estivale du laboratoire. Les pilotes ont été maintenus en fonctionnement habituel, en continu, avec une surveillance régulière, mais aucune analyse n'a été réalisée. D'après la législation française (MEEDDM, 2009), les seuils des deux paramètres réglementaires en sortie de système sont de $30 \mathrm{mg} \cdot \mathrm{L}^{-1}$ pour les MES et de $35 \mathrm{mg} \mathrm{O} \cdot \mathrm{L}^{-1}$ pour la $\mathrm{DBO}_{5}$. Ces paramètres sont stables tout en étant très inférieurs au seuil imposé (Figures 3a, 3b). L'évolution de l'épuration de la DCO est lente mais constante (Figure 3c), sans que cela n'ait une influence importante sur la $\mathrm{DBO}_{5}$. Ce phénomène est retrouvé aussi dans les filtres à coco (EPNAC, 2014), où un relargage de tanins est associé à des concentrations en DCO plus élevées en sortie de station (rendements autour de $60 \%$ ), mais sans effet sur la $\mathrm{DBO}_{5}$. L'évolution du $\mathrm{pH}$ souligne que les pilotes chènevotte sur grignons d'olives ont tendance à présenter un $\mathrm{pH}$ neutre, tandis que l'eau de sortie du pilote copeaux de coco est plutôt acide (Figure 3d). L'ensemble des pilotes montre un rendement d'élimination moyen de MES supérieur à $90 \%$ et une réduction en $\mathrm{DBO}_{5}$ de plus de $96 \%$; en termes d'épuration, les pilotes chènevotte sur grignons d'olives ont une capacité similaire à celle du pilote copeaux de coco. 

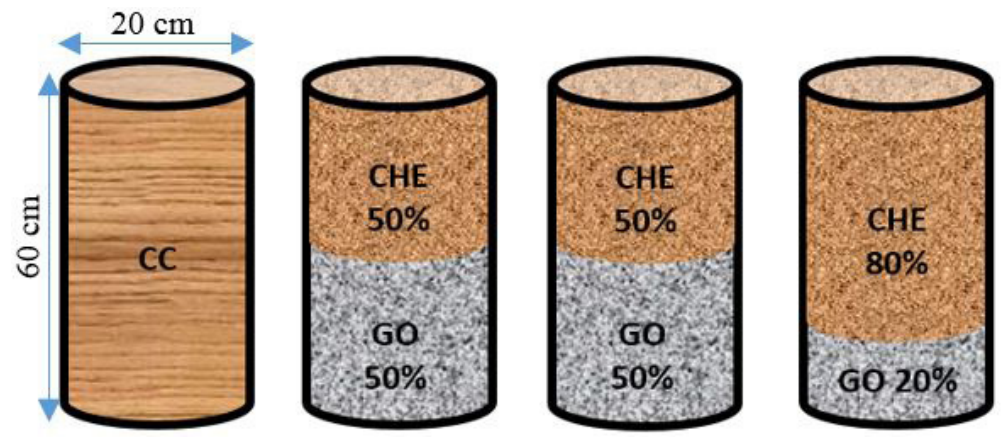

Figure 1. Pilotes de biofiltration : $\mathrm{CC}$ : copeaux de coco; $\mathrm{CHE}$ : chènevotte; GO : grignons d'olives.

Biofiltration pilots: CC: coconut husks; CHE: hemp shives; GO: olive pomace.

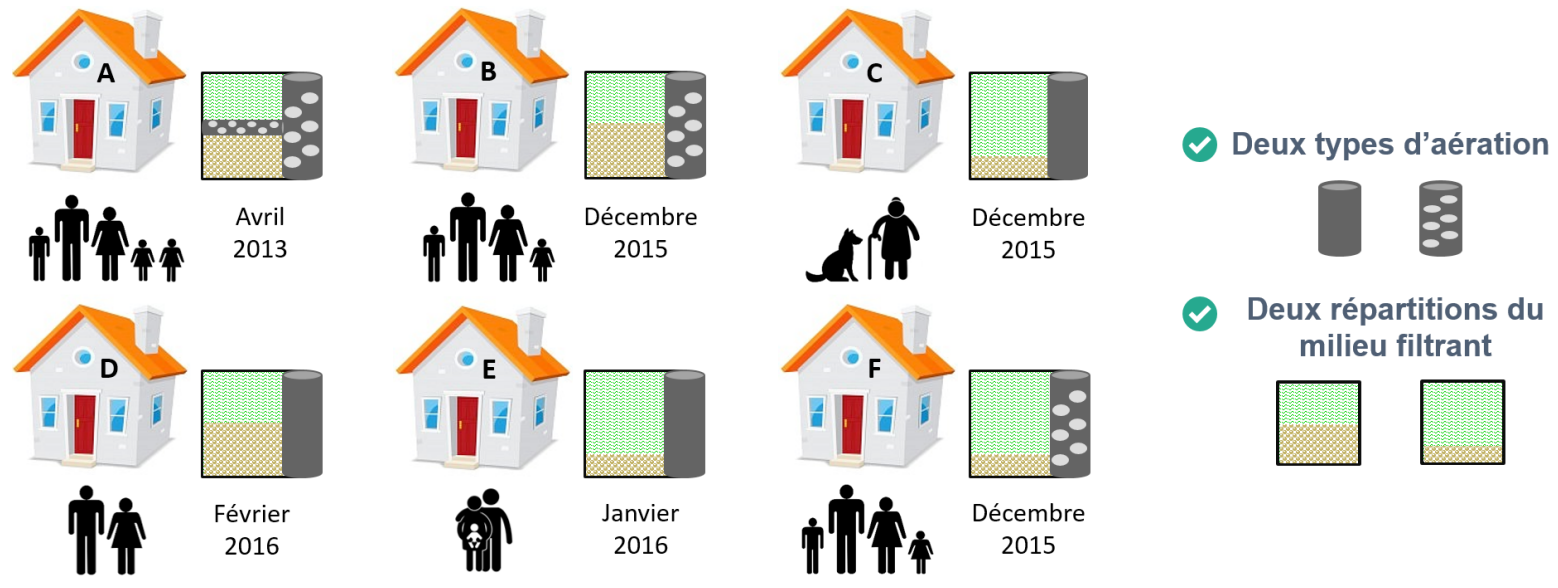

Figure 2. Conditions opératoires de chaque site. Sites' operating conditions.

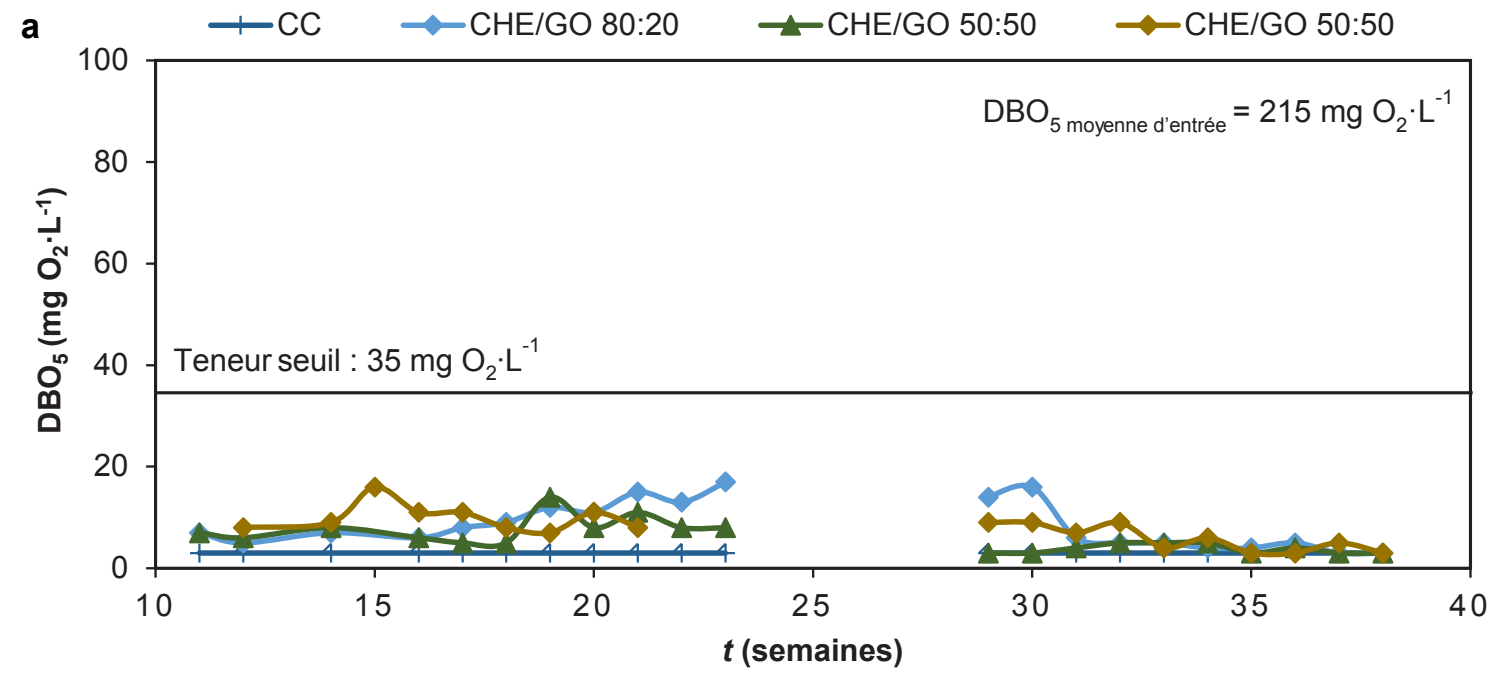

Figure 3. Paramètres environnementaux de l'eau en sortie des pilotes : a) demande biochimique en oxygène en cinq jours $\left(\mathrm{DBO}_{5}\right)$. CC : copeaux de coco; $\mathrm{CHE}$ : chènevotte; $\mathrm{GO}$ : grignons d'olives.

Environmental parameters of the pilots' outlet water: a) biochemical oxygen demand at five days $\left(\mathrm{BOD}_{5}\right)$. CC: coconut husks; CHE: hemp shives; GO: olive pomace. 

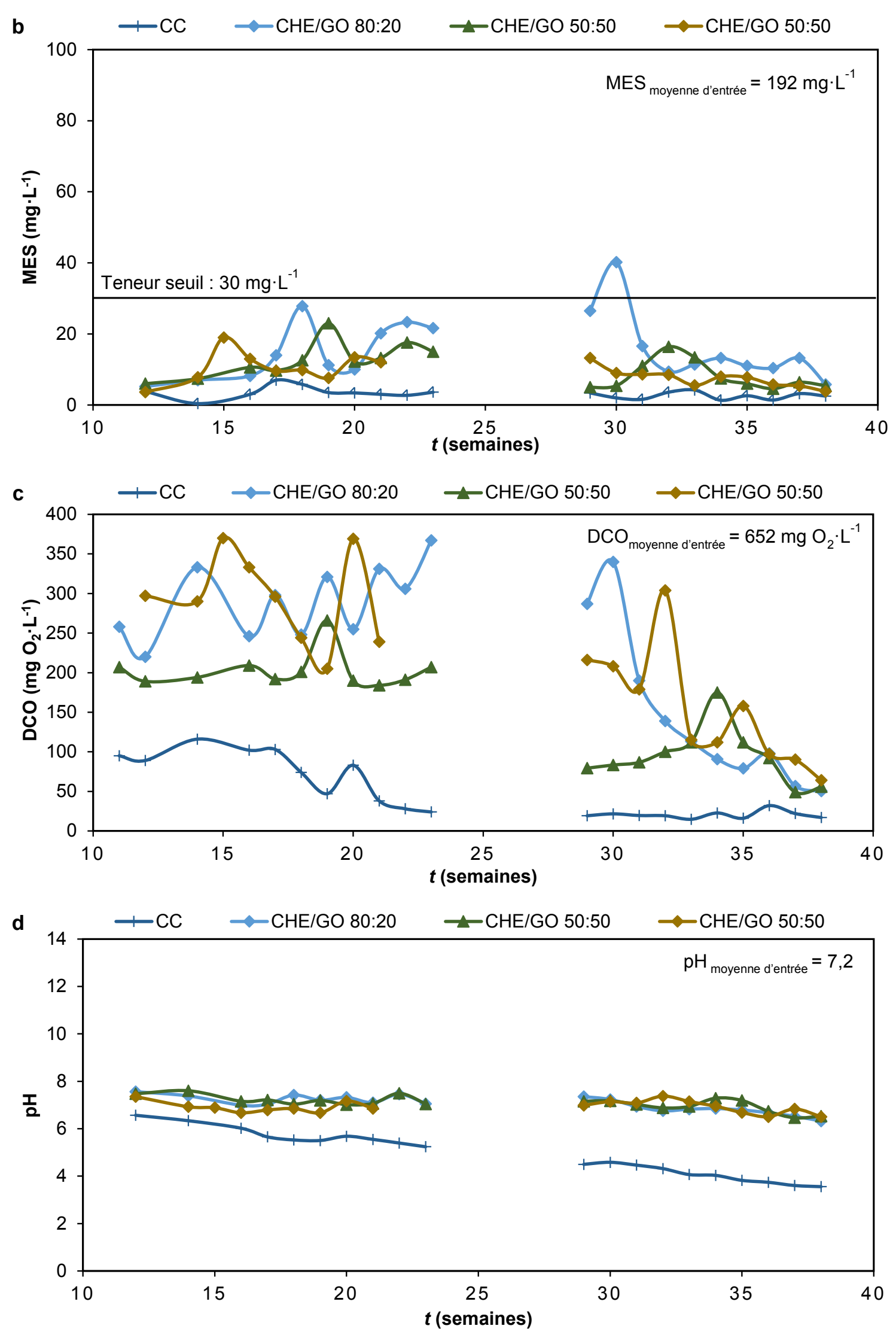

Figure 3. Paramètres environnementaux de l'eau en sortie des pilotes : b) matières en suspension (MES), (suite) c) demande chimique en oxygène (DCO) et d) $\mathrm{pH}$. $\mathrm{CC}$ : copeaux de coco; $\mathrm{CHE}$ : chènevotte; GO : grignons d'olives.

(continued) Environmental parameters of the pilots' outlet water: b) total suspended solids (TSS), c) chemical oxygen demand (COD), and d) pH. CC: coconut husks; CHE: hemp shives; GO: olive pomace. 
L'ensemble de ces résultats indique d'une part que la mise en régime (développement de la biomasse épuratrice) est inférieure ou égale à dix semaines, d'autre part, les performances épuratoires conduisent à des valeurs des paramètres réglementaires inférieurs aux seuils, ce qui est de bon augure pour les expérimentations terrains.

\subsection{Etude terrain}

Les figures $4 \mathrm{a}, 4 \mathrm{~b}$ et $4 \mathrm{c}$ montrent l'évolution de la $\mathrm{DBO}_{5}$, de MES et de la DCO respectivement, au cours de 600 jours de fonctionnement ininterrompu. Ces graphes sont tracés à partir de la date de mise en route des installations. Certains sites fonctionnent mieux que d'autres, notamment les sites B, $\mathrm{C}$ et $\mathrm{E}$ qui ont le meilleur démarrage. En réalité, au tout début des opérations, des problèmes techniques sont survenus sur ces trois sites : le biofiltre du site B a été alimenté pendant les premiers 90 jours par des eaux usées brutes (EUB). En effet, à cause d'un mauvais raccordement, les EUB ont été délivrées directement sur la surface du biofiltre sans passer en amont par la fosse toutes eaux (cuve en béton de 3000 L). Cette erreur technique semble être à l'origine d'un démarrage plus rapide de l'activité de la flore bactérienne, comme si un ensemencement bactérien avait été bénéfique. Le biofiltre du site C, par un dysfonctionnement du système de pompage survenu à 120 jours, a été submergé de façon ponctuelle, mais intense par un volume important d'EUPT. Enfin, le biofiltre du site E, a été complètement immergé pendant les 30 premiers jours d'opération à cause d'une absence de pompage du poste de relevage situé en aval. La rapidité avec laquelle ces biofiltres ont développé une bonne performance épuratrice semble être liée à ces imprévus, qui dans tous les cas ont conduit à un mouillage rapide et global du massif filtrant.

De plus, afin de permettre une meilleure répartition des eaux à la surface du biofiltre, une scarification a été réalisée autour des 300 jours (TALBOT et al., 1996). Ceci induit donc à une réoxygénation des filtres tout en restituant leurs pleines capacités d'infiltration. Les résultats de cette opération ont en particulier une répercussion dans le site $\mathrm{D}$, où une amélioration de sa performance est remarquée.

Pour le site A, dans un premier temps les données sont disponibles jusqu'à 340 jours, puis, comme le montre la figure $4 \mathrm{~d}$, entre la troisième et quatrième année de fonctionnement. Ce dernier graphe permet ainsi de constater une efficacité remarquable à long terme. En effet, ce site qui fonctionne en continu depuis plus de quatre ans, montre des valeurs de $\mathrm{DBO}_{5}$ et de MES stationnaires et bien au-dessous des limites réglementaires. Le suivi de ce site permettra d'établir la longévité de ce milieu filtrant. De plus, pour la DCO, il présente des valeurs majoritairement entre 80 et $100 \mathrm{mg} \mathrm{O} \cdot \mathrm{L}^{-1}$, ce qui est conforme à ce qui est attendu pour ce type d'installation. En somme, les pourcentages moyens de réduction de la DCO, de la $\mathrm{DBO}_{5}$ et de MES pour ce site sont respectivement de plus de $83 \%, 97 \%$ et $96 \%$. Les rendements d'élimination moyens en MES pour les sites B, C et E sont de $82 \%, 80 \%$ et $79 \%$ respectivement. Pour la $\mathrm{DBO}_{5}$, ce rendement est de $89 \%$, $87 \% 89 \%$, pour les mêmes sites. Des rendements similaires ont été trouvés pour des filtres à sable et à copeaux de coco (OLIVEIRA CRUZ et al., 2013; EFFERT et al., 1984).

La figure 5 propose une synthèse des résultats de la figure 4, de façon à comparer la durée nécessaire par chacun des sites pour arriver aux valeurs cibles en fonction des conditions initiales; tout en tenant compte des différents problèmes techniques et/ ou modifications mécaniques survenus.

Comme dit précédemment, les sites $\mathrm{B}, \mathrm{C}$ et $\mathrm{E}$, qui ont subi une immersion accidentelle, semblent avoir une dynamique de mise en régime plus rapide, et ce d'autant plus avec la répartition 80:20. Par contre, aucun lien évident ne peut pas être établi entre les performances et la présence de tubes avec ou sans aération, pas plus qu'avec les proportions envisagées de hauteur de matériaux ou le nombre d'habitants. Une faible hauteur de la couche de support pour une hauteur majeure de la couche active sera donc à privilégier.

Une différence importante est aperçue lorsqu'on compare les résultats obtenus dans l'étude pilote avec ceux obtenus dans l'étude terrain. En effet, le temps de mise en régime est supérieur dans l'étude terrain, ceci est surement lié à la variabilité de la qualité des eaux usées domestiques d'entrée, que ce soit entre les différentes installations ou au sein d'une même installation au cours du temps.

\section{CONCLUSIONS}

Vu la qualité des eaux traitées et les valeurs obtenues pour les paramètres réglementaires à l'issue des campagnes d'expérience terrain d'environ 600 jours, le milieu chènevotte sur grignons d'olives, dans des installations telles que dimensionnées, se présente comme une alternative originale et fiable aux copeaux de coco. La distribution 80:20 permet une performance correcte tout en utilisant moins de grignons d'olives.

En outre, il semble qu'un mouillage initial du biofiltre avec des eaux usées ou prétraitées pourrait permettre un démarrage plus rapide du processus d'épuration. 

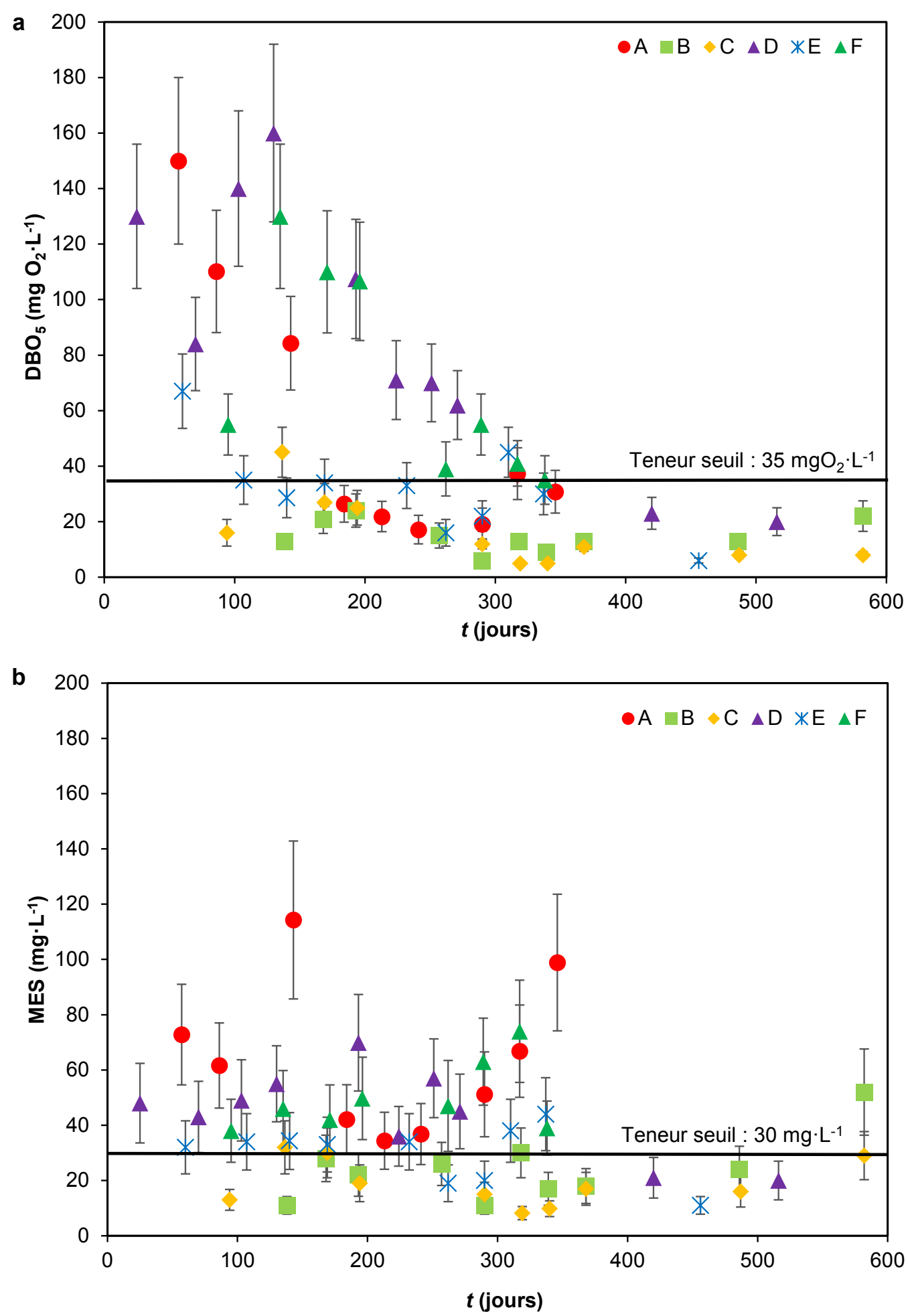

Figure 4. Paramètres environnementaux de l'eau en sortie des prototypes terrain : a) demande biochimique en oxygène en cinq jours $\left(\mathrm{DBO}_{5}\right)$ et $\mathrm{b}$ ) matières en suspension (MES).

Environmental parameters of the full scale prototypes' outlet water: a) biochemical oxygen demand at five days $\left(\mathrm{BOD}_{5}\right)$, and b) total suspended solids (TSS). 

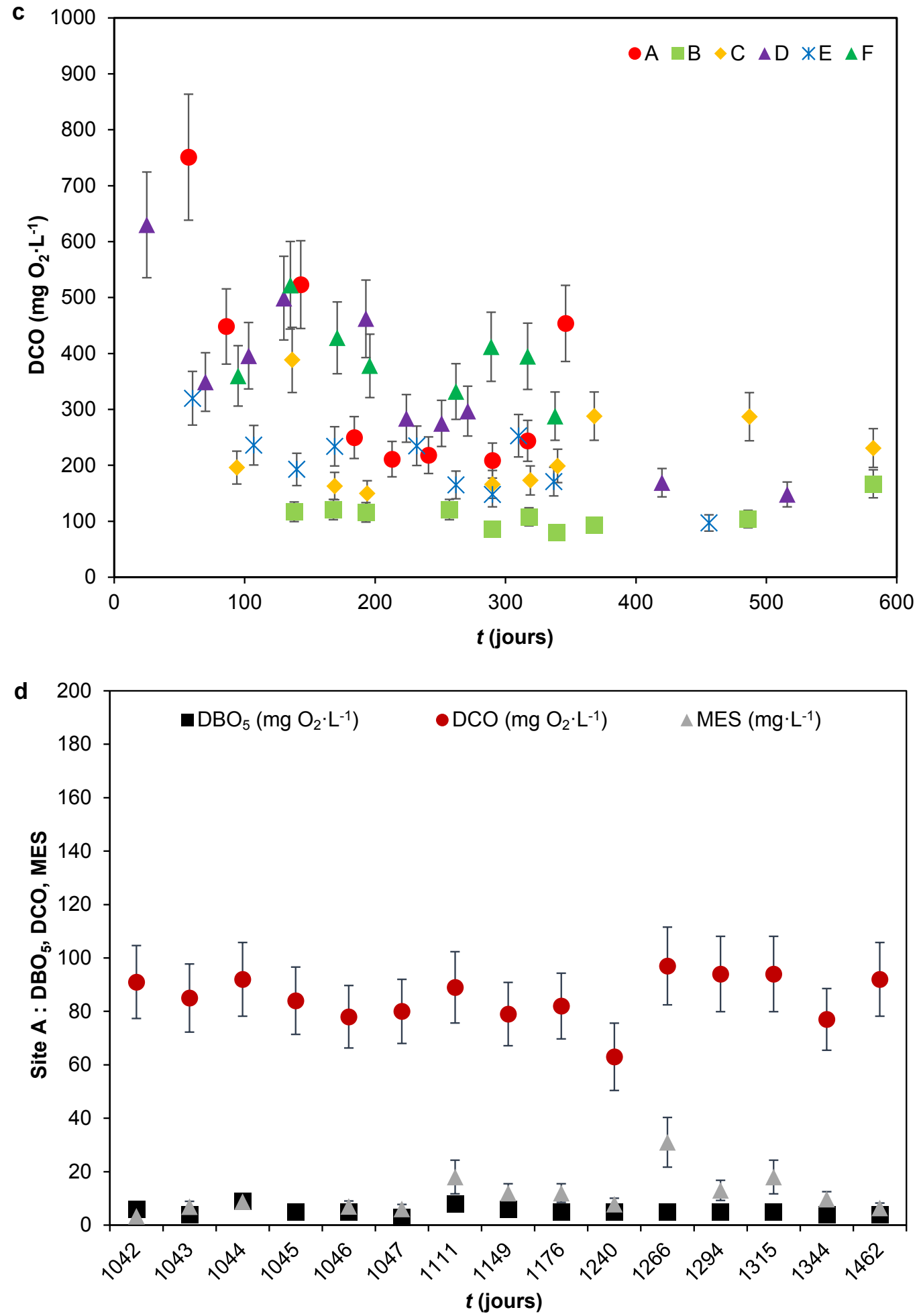

Figure 4. Paramètres environnementaux de l'eau en sortie des prototypes terrain : c) demande chimique (suite) en oxygène (DCO) et $\mathrm{d}$ ) résultats du site $\mathrm{A}$ après trois ans de fonctionnement.

(continued) Environmental parameters of the full scale prototypes' outlet water: c) chemical oxygen demand (COD), and d) site A results after three years of continuous operation. 
Durée (jours)

d'atteinte du seuil de

$\mathrm{DBO}_{5} \quad \mathrm{MES} \quad$ Répartition Aération

$\begin{array}{ccc}\begin{array}{c}\text { Nombre } \\ \text { d'habitants }\end{array} & \begin{array}{c}\text { Mouillage du } \\ \text { milieu filtrant }\end{array} & \begin{array}{c}\text { Amélioration } \\ \text { après scarification }\end{array}\end{array}$

A 180
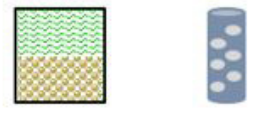

B $<140<140$
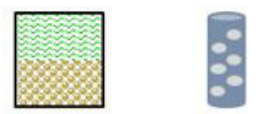

\|\|$_{* 4}$

thit

Oui

$=$

C $\quad<90<90$
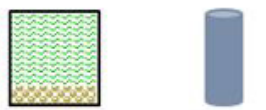

Aी

Oui

$=$

D $\quad 420$

420
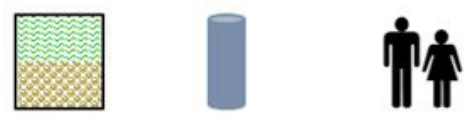

Oui

E $\quad 100 \quad 60$
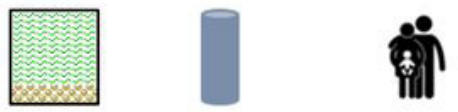

Oui

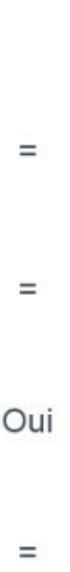

F $\quad 340$

340

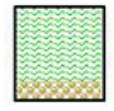

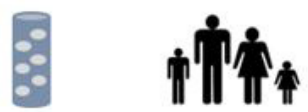

Figure 5. Récapitulatif de l'étude terrain. Summary of the field study.

\section{REMERCIEMENTS}

Les auteurs remercient l'Association Nationale de la Recherche et de la Technologie (ANRT) pour sa contribution à ces travaux (convention CIFRE N²015/0062).

\section{RÉFÉRENCES BIBLIOGRAPHIQUES}

ASSOCIATION FRANÇAISE DE NORMALISATION (AFNOR) (1998). Qualité de l'eau - Détermination de la demande biochimique en oxygène après $n$ jours (DBOn). Norme française NF EN 1899-1, France.

ASSOCIATION FRANÇAISE DE NORMALISATION

(AFNOR) (2001). Qualité de l'eau - Détermination du $p H$. Norme française NF T90-008, France.

ASSOCIATION FRANÇAISE DE NORMALISATION

(AFNOR) (2005). Qualité de l'eau - Dosage des matières en suspension. Norme française NF EN 872, France.

BERLANDJ.-M. (2012). Techniques etgestion de l'assainissement non collectif - Filières de traitement. Techniques de l'Ingénieur, France, 14 p.

BRETON A. (2013). Potentialité de la biomasse végétale pour le traitement des eaux usées domestiques : développement d'un procédé de biofiltration pour l'assainissement non collectif. Thèse de doctorat, Univ. Toulouse, France, 292 p.

CAUCHI A. et C. VIGNOLES (2011). Petites installations d'assainissement. Éditions TECHNIP, France, 416 p.

CHABAUD S. (2007). Influence du biofilm sur les performances des systèmes de traitement par infiltration dans le sol: application à l'assainissement non collectif. Thèse de doctorat, Univ. Nantes, France, 193 p.

DUBOIS V. et C. BOUTIN (2017). Comparison of the design criteria of 141 onsite wastewater treatment systems available on the French market. J. Environ. Manage., 216, 299-304.

EFFERT D., J. MORAND et M. CASHELL (1984). Field performance of three onsite effluent polishing units. Dans : Proceedings of the Fourth National Symposium on Individual and Small Community Sewage Systems. New Orleans (LA), États-Unis, pp. 7-85.

ÉVALUATION DES PROCÉDÉS NOUVEAUX D'ASSAINISSEMENT DES PETITES ET MOYENNES COLLECTIVITÉS (EPNAC) (2014). Filtres à coco pour le petit collectif-Premier Tech. Veille des procédés nouveaux, Office nationale de l'eau et des milieux aquatiques, France, 10 p. 
GALLIEN P., A. BRETON, C. VIALLE, M. VIGNOLES, C. ALBASI, C. SABLAYROLLES et G. VILAREM (2016). Dispositif et procédé d'évaluation de la capacité d'un matériau solide à constituer un milieu filtrant support de biomasse épuratrice pour l'épuration d'eaux usées. FR 3036 394, France, 42 p.

GARZON-ZUNIIGA M.A., G. BUELNA et G.E. MOELLERCHÀVEZ (2012). La biofiltración sobre materiales orgánicos, nueva tecnología sustentable para tratar agua residual en pequeńas comunidades e industrias. Tecnol. Cienc. Agua, 3, 153-161.

LENS P.N., P.M. VOCHTEN, L. SPELEERS et W.H. VERSTRAETE (1994). Direct treatment of domestic wastewater by percolation over peat, bark, and woodchips. Water Res., 28, 17-26.

MINISTÈRE DE L'ÉCOLOGIE, DE L'ÉNERGIE, DU DÉVELOPPEMENT DURABLE ET DE LA MER (MEEDDM) (2009). Arrêté du 7 septembre 2009 fixant les prescriptions techniques applicables aux installations d'assainissement non collectif recevant une charge brute de pollution organique inférieure ou égale à $1,2 \mathrm{~kg} / \mathrm{j}$ de $D B O_{5}$. https://www.legifrance.gouv.fr/affich Texte.do?cidTexte=L EGITEXT000021125886 (consultation le 28 août 2016.).
OLIVEIRA CRUZ de L.M., R. STEFANUTTI, B. CORAUCCI FILHO et A.L. TONETTI (2013). Coconut shells as filling material for anaerobic filters. SpringerPlus, 2, 655.

ORGANISATION INTERNATIONALE DE NORMALISATION (ISO) (2002). Qualité de l'eau Détermination de l'indice de demande chimique en oxygène (ST-DCO). ISO 15705:2002.

ORGANISATION MONDIALE DE LA SANTÉ et FONDS DES NATIONS UNIES POUR L'ENFANCE (OMS/ UNICEF) (2013). Joint monitoring programme for water supply and sanitation. ISBN 978924150539 0, 40 p.

TALBOT P., G. BÉLANGER, M. PELLETIER, G. LALIBERTÉ et Y. ARCAND (1996). Development of a biofilter using an organic medium for on-site wastewater treatment. Water Sci. Technol., 34, 435-441. 